\title{
Validation of a dynamically adaptive lattice Boltzmann method for 2D thermal convection simulations
}

\author{
Kai Feldhusen ${ }^{1,2}$, Ralf Deiterding ${ }^{1}$, and Claus Wagner ${ }^{1,2}$
}

\begin{abstract}
Utilizing the Boussinesq approximation, a double-population thermal lattice Boltzmann method (LBM) for forced and natural convection in two space dimensions is developed and validated. A block-structured dynamic adaptive mesh refinement procedure tailored for LBM is applied to enable computationally efficient simulations of high Rayleigh number configurations which are characterized by a large scale disparity in boundary layers and free stream flow. As test cases, the analytically accessible problem of a two-dimensional (2D) forced convection flow through two porous plates and the non-Cartesian configuration of a heated rotating cylinder are considered. The effectiveness of the overall approach is demonstrated for the $2 \mathrm{D}$ natural convection benchmark of a cavity with differentially heated walls at Rayleigh numbers from $10^{3}$ up to $10^{8}$.
\end{abstract}

Keywords-Lattice Boltzmann method, thermal convection, adaptive mesh refinement

\section{INTRODUCTION}

In recent years, the lattice Boltzmann method has emerged as a powerful alternative to traditional Navier-Stokes (NS) solvers [1]. Instead of discretizing the NS equations directly, the LBM is based on solving a simplified version of the Boltzmann equation in a specially chosen discrete phase space. Using a Chapman-Enskog expansion, it can be shown that the approach recovers the NS equations in the limit of a vanishing Knudsen number [2]. Originally proposed for the isothermal weakly compressible case, several method enhancements for incompressibility [3], [4] as well as incorporation of a buoyancy-driven temperature

\footnotetext{
${ }^{1}$ German Aerospace Center (DLR), Institute of Aerodynamics and Flow Technology, Bunsenstr. 10, 37073 Göttingen, Germany email: kai.feldhusen@dlr.de.

${ }^{2}$ Technische Universität Ilmenau, Institute of Thermo- and Fluiddynamics, 98693 Ilmenau
}

field for thermal convection flows are available [5], [6]. Here, we have chosen to pursue the strictly incompressible double distribution function (DDF) approach proposed by Guo et al. [7].

While the original LBM is formulated on a uniform Cartesian grid, an increase of local resolution is particularly desirable in the thermal boundary layers close to heated objects and walls. So far, the majority of DDF LBM methods with on-the-fly mesh adaptation has been proposed for isothermal two-phase flows, cf. [8]. Kuznik et al. [9] demonstrated the computational benefit of a non-uniform grid for a thermal DDF LBM method; yet, their approach is restricted to purely Cartesian domains. Our objective is to close this gap by incorporating a DDF LBM method into a block-based dynamic adaptive mesh refinement (AMR) method [10].

The outline of this paper is as follows: In Section II we discuss the details of the numerical method, including the adopted thermal lattice Boltzmann approach, the block-based AMR method and the treatment of geometrically complex boundaries in the originally Cartesian scheme. Section III presents the computational results, where the analytic validation example of the $2 \mathrm{D}$ flow between two moving porous plates, the 2D flow around a rotating heated cylinder and the wellknown benchmark case of a two-dimensional cavity with differentially heated walls are considered. The conclusions including a short outlook are given in Section IV.

\section{NUMERICAL METHOD}

\section{A. Thermal lattice Boltzmann scheme}

The incompressible two-dimensional LBM constructed under Boussinesq approximation used in the present work has been proposed by Guo et al. [7]. Note that the extensions to three dimensions is 


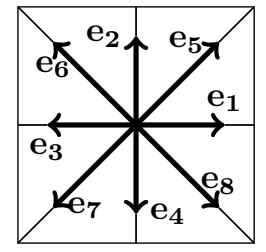

Fig. 1. Numerical stencil of D2Q9 - Discrete velocity directions in a computational cell

straightforward, cf. [11]. By using the BhatnagarGross-Krook (BGK) collision model [12], the lattice Boltzmann equation for the partial probability distribution function $f_{i}$ with force field term $F_{i}$ can be formulated as

$$
\begin{aligned}
& f_{i}\left(\mathbf{x}+c \mathbf{e}_{i} \Delta t, t+\Delta t\right)=f_{i}(\mathbf{x}, t) \\
& \quad-\frac{1}{\tau_{\nu}}\left(f_{i}(\mathbf{x}, t)-f_{i}^{(e q)}(\mathbf{x}, t)\right)+\Delta t F_{i} .
\end{aligned}
$$

In the DDF approach, a set of corresponding lattice Boltzmann equations

$$
\begin{aligned}
& g_{i}\left(\mathbf{x}+c \mathbf{e}_{i} \Delta t, t+\Delta t\right)= \\
& \quad g_{i}(\mathbf{x}, t)-\frac{1}{\tau_{\mathcal{D}}}\left(g_{i}(\mathbf{x}, t)-g_{i}^{(e q)}(\mathbf{x}, t)\right)
\end{aligned}
$$

is introduced for distributions $g_{i}$ that are used to convect the macroscopic scalar quantity, here temperature, with the flow field. In the latter, $\mathbf{e}_{i}$ is the unit velocity vector in direction of the $i$ th discrete velocity space direction, $t$ and $\Delta t$ denote the time and time step, $x$ the position, $\Delta x$ the spatial increment, and $c=\Delta x / \Delta t$ is the particle speed. The relaxations times are $\tau_{\nu}$ for the flow field and $\tau_{\mathcal{D}}$ for the temperature field. The respective equilibrium distribution functions are denoted by $f_{i}^{(e q)}$ and $g_{i}^{(e q)}$. In the two-dimensional case, a model with nine discrete unit velocities is realized for simulating the flow field (D2Q9) and an operator with four discrete velocities for simulating the temperature field (D2Q4). The orientation of the discrete unit length velocity vectors $\mathbf{e}_{i}$ are depicted in Fig. 1.

The basic LBM algorithm is divided into the steps of transport (or streaming) and collision that are applied basically identically to (1) and (2). The transport step represents the advection of fluid particles along the corresponding discrete velocities and reads

$$
\mathcal{T}: \quad \tilde{f}_{i}\left(\mathbf{x}+c \mathbf{e}_{i} \Delta t, t+\Delta t\right)=f_{i}(\mathbf{x}, t) .
$$

Relaxing the distribution functions towards the local equilibrium is performed on the transported distribution functions in the collision step

$$
\begin{gathered}
\mathcal{C}: \quad f_{i}\left(\mathbf{x}+c \mathbf{e}_{i} \Delta t, t+\Delta t\right)=\tilde{f}_{i}\left(\mathbf{x}+c \mathbf{e}_{i} \Delta t\right. \\
t+\Delta t)-\frac{1}{\tau_{\nu}}\left(\tilde{f}_{i}(\mathbf{x}, t)-\tilde{f}_{i}^{(e q)}(\mathbf{x}, t)\right)
\end{gathered}
$$

With the pressure $p$ and the velocity $\mathbf{u}$ as independent variables, the specific equilibrium distribution function $f_{i}^{(e q)}$ is defined as [7]

$$
f_{i}^{(e q)}= \begin{cases}-4 \sigma \frac{p}{c^{2}}-s_{i}(\mathbf{u}), & \text { for } i=0, \\ \lambda \frac{p}{c^{2}}+s_{i}(\mathbf{u}), & \text { for } i=1, \ldots, 4 \\ \gamma \frac{p}{c^{2}}+s_{i}(\mathbf{u}), & \text { for } i=5, \ldots, 8\end{cases}
$$

where the parameters $\sigma, \lambda$, and $\gamma$ satisfy $\lambda+\gamma=\sigma$ and $\lambda+2 \gamma=1 / 2$. The functions $s_{i}(\mathbf{u})$ depend on the macroscopic velocity vector $\mathbf{u}$ and the discrete velocity vector $\mathbf{e}_{i}$ and obey

$$
s_{i}(\mathbf{u})=\omega_{i}\left[3 \frac{\mathbf{e}_{i} \cdot \mathbf{u}}{c}+4.5 \frac{\left(\mathbf{e}_{i} \cdot \mathbf{u}\right)^{2}}{c^{2}}-1.5 \frac{|\mathbf{u}|^{2}}{c^{2}}\right],
$$

where the coefficients are given by $\omega_{0}=$ $4 / 9, \omega_{1, \ldots, 4}=1 / 9$, and $\omega_{5, \ldots, 8}=1 / 36$. Using (5) and (6), the macroscopic values for velocity and dynamic pressure are given as

$$
\mathbf{u}=\sum_{i=1}^{8} c \mathbf{e}_{i} f_{i}, \quad p=\frac{c^{2}}{4 \sigma}\left[\sum_{i=1}^{8} f_{i}+s_{0}(\mathbf{u})\right]
$$

For the temperature field, the equilibrium function $g_{i}^{(e q)}$ reads

$$
g_{i}^{(e q)}=\frac{T}{4}\left[1+2 \frac{\mathbf{e}_{i} \cdot \mathbf{u}}{c}\right], \text { for } i=1, \ldots, 4 .
$$

and the mascroscopic temperature is $T=\sum_{i=1}^{4} g_{i}$. Since the fluid is assumed to be incompressible, a linear dependency between temperature differences and gravitational forces is applied (Boussinesq approximation), cf. [13], and used to define the force term $F_{i}$. The force acts only in the two direct vertical directions with indices 2 and 4 according to Fig. 1 [7] and is given here by

$$
F_{i}=\frac{1}{2}\left(\delta_{i 2}+\delta_{i 4}\right) \mathbf{e}_{i} \cdot \mathbf{F}
$$


with

$$
\mathbf{F}=\mathbf{g} \beta\left(T-T_{r e f}\right),
$$

where $\mathrm{g}$ and $\beta$ are the acceleration vector of gravity and the coefficient of thermal expansion, respectively; $T_{r e f}$ is the average temperature. The force term establishes the coupling between the lattice Boltzmann equations for the flow field (1) and the temperature field (2).

Note that through a multi-scale ChapmanEnskoq expansion, the incompressible NavierStokes equations can be derived from the discussed incompressible LBGK model. After neglecting the viscous heat dissipation and compression work carried out by the pressure, the temperature field obeys a passive scalar equation. In sum, the approximated incompressible equations in this work are, cf. [7],

$$
\begin{aligned}
\nabla \cdot \mathbf{u} & =0 \\
\frac{\partial \mathbf{u}}{\partial t}+\nabla \cdot(\mathbf{u u}) & =-\nabla p+\nu \nabla^{2} \mathbf{u}+\mathbf{F} \\
\frac{\partial T}{\partial t}+\nabla \cdot(\mathbf{u} T) & =\mathcal{D} \nabla^{2} T
\end{aligned}
$$

The kinematic viscosity $\nu$ and the thermal diffusivity $\mathcal{D}$ are related to the dimension-less collision times by $\nu=\frac{1}{6}\left(2 \tau_{\nu}-1\right) c \Delta x$ and $\mathcal{D}=$ $\frac{1}{4}\left(2 \tau_{\mathcal{D}}-1\right) c \Delta x$. Introducing the physical speed of sound as $c_{s}=c / \sqrt{3}$ these expressions yield the relations

$$
\tau_{\nu}=\frac{\nu+c_{s}^{2} \Delta t / 2}{c_{s}^{2} \Delta t}, \quad \tau_{\mathcal{D}}=\frac{\mathcal{D}+\frac{3}{2} c_{s}^{2} \Delta t / 2}{\frac{3}{2} c_{s}^{2} \Delta t},
$$

which can be used to evaluate the dimensional-less collision times in (1) and (2) for given macroscopic gas properties $\nu, \mathcal{D}$ and time step $\Delta t$.

\section{B. Adaptive mesh refinement}

For local dynamic mesh adaptation we have adopted the block-structured AMR method after Berger \& Collela [14]. This method was orginally designed for time-explicit finite volume schemes, however, its recursive execution procedure and natural consideration of time step refinement make it equally applicable to lattice Boltzmann schemes. In order to fit smoothly into our existing, fully parallelized finite volume AMR software system AMROC [10], we have implemented the LBM cellbased. In the block-based AMR approach, finite volume cells are clustered with a special algorithm

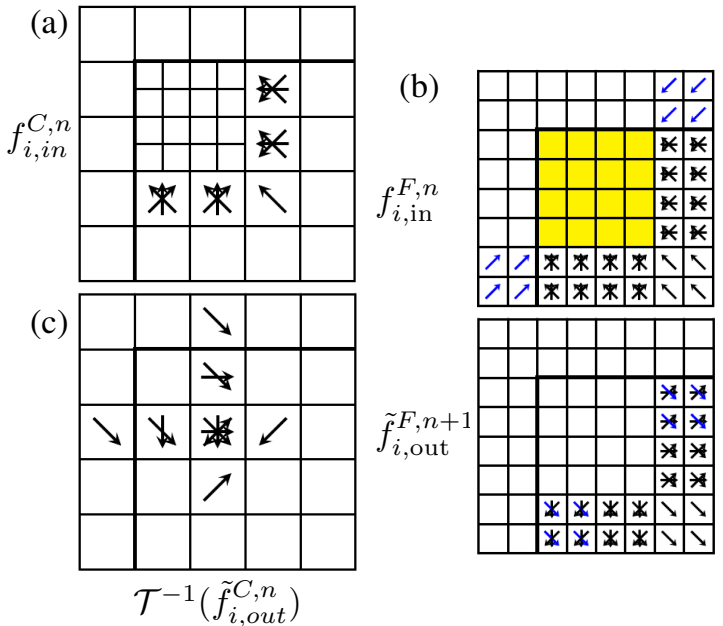

Fig. 2. Visualization of distributions involved in data exchange at a coarse $(C)$ - fine $(F)$ boundary. The thick black lines indicate a physical boundary. (a) Coarse distributions going into fine grid; (b) ingoing interpolated fine distributions in halos (top), outgoing distributions in halos after two fine-level transport steps (bottom); (c) averaged distributions replacing coarse values before update is repeated in cells next to boundary.

into non-overlapping rectangular grids. The grids have a suitable layer of halo cells for synchronization and applying inter-level and physical boundary conditions. Refinement levels are integrated recursively. With index $l$ denoting the AMR level, the spatial mesh width $\Delta x_{l}$ and the time step $\Delta t_{l}$ are refined by the same factor $r_{l}$, where we assume $r_{l} \geq 2$ for $l>0$ and $r_{0}=1$. In the adaptive thermal LBM, it is of foremost importance that the dimension-less collision times of the DDF LBM are adjusted on a level basis according to (12) as the time step is recursively refined. In addition to this, the interface region requires a specialized treatment to ensure consistent transport of coarsegrid distributions into refined cells and of fine-grid distributions into the coarse cells adjacent to the refinement boundary. Since the D2Q4 stencil is just a simplified version of the D2Q9 method, we restrict our description of the interface algorithm to the latter. Distinguishing between the transport and collision operators, $\mathcal{T}$ and $\mathcal{C}$, cf. (3) and (4), the steps of our method for a refinement factor of 2 are:

1) Complete update on coarse grid: $f_{i}^{C, n+1}:=$ $\mathcal{C} \mathcal{T}\left(f_{i}^{C, n}\right)$

2) Use coarse grid distributions $f_{i, \text { in }}^{C, n}$ that prop- 
agate into the fine grid, cf. Fig. 2(a), to construct initial fine grid halo values $f_{i, \text { in }}^{F, n}$, cf. Fig. 2(b).

3) Complete transport $\tilde{f}_{i}^{F, n}:=\mathcal{T}\left(f_{i}^{F, n}\right)$ on whole fine mesh. Collision $f_{i}^{F, n+1 / 2}:=$ $\mathcal{C}\left(\tilde{f}_{i}^{F, n}\right)$ is applied only in the interior cells (yellow in Fig. 2(b)).

4) Repeat 3. to obtain $\tilde{f}_{i}^{F, n+1 / 2}:=$ $\mathcal{T}\left(f_{i}^{F, n+1 / 2}\right)$ and $f_{i}^{F, n+1}:=\mathcal{C}\left(\tilde{f}_{i}^{F, n+1 / 2}\right)$.

5) Average outgoing distributions from fine grid halos (Fig. 2(c)), that is $\tilde{f}_{i, \text { out }}^{F, n+1 / 2}$ in the inner halo layer and $\tilde{f}_{i \text {,out }}^{F, n}$ (outer halo layer) to obtain $\tilde{f}_{i, \text { out }}^{C, n}$.

6) Revert transport for averaged outgoing distributions, $\bar{f}_{i \text {,out }}^{C, n}:=\mathcal{T}^{-1}\left(\tilde{f}_{i \text {,out }}^{C, n}\right)$, and overwrite those in the previous coarse grid time step, cf. Fig. 2(d).

7) Synchronization of $f_{i}^{C, n}, \bar{f}_{i, \text { out }}^{C, n}$ on entire level.

8) Repeat complete update on coarse grid cells next to coarse-fine boundary only: $f_{i}^{C, n+1}:=\mathcal{C} \mathcal{T}\left(f_{i}^{C, n}, \bar{f}_{i, \text { out }}^{C, n}\right)$

In this description and in Fig. 2, the time steps on the coarse level $C$ are indexed by the superscript $n$, index $F$ denotes the fine level and the subscripts in and out indicate distributions streaming in- and outwards of the fine grid along the coarse-fine boundary. The overall algorithm is computationally equivalent to the method by Chen et al. [15] but explicitly tailored to the Berger-Collela recursion that updates coarse grids in their entirety before fine grids are computed.

\section{Curved boundary handling}

We represent non-Cartesian boundaries implicitly on the adaptive Cartesian grid by utilizing a scalar level set function $\varphi$ that stores the distance to the boundary surface. The boundary surface is located exactly at $\varphi=0$ and the boundary outer normal in every mesh point can be evaluated as $\mathbf{n}=-\nabla \varphi /|\nabla \varphi|$, [10]. We treat a fluid cell as an embedded ghost cell if its midpoint satisfies $\varphi<0$.

In order to implement non-Cartesian boundary conditions with the LBM, we have chosen to pursue for now a 1st order accurate ghost fluid approach. In our technique, the density distributions in embedded ghost cells are adjusted to model the boundary conditions of a non-Cartesian reflective wall moving with velocity vector $\mathbf{w}$ before applying the unaltered LBM. The last step involves interpolation and mirroring of $p, T, \mathbf{u}$, across the boundary to $p^{\prime}, T^{\prime}$ and $\overline{\mathbf{u}}$ and modification of the macroscopic velocity vector in the immersed boundary cells to $\mathbf{u}^{\prime}=2 \mathbf{w}-\overline{\mathbf{u}}$, cf. [10]. From the newly constructed macroscopic values the distributions in the embedded ghost cells are simply set to $f_{i}^{e q}\left(p^{\prime}, \mathbf{u}^{\prime}\right)$ and $g_{i}^{e q}\left(T^{\prime}\right)$.

\section{RESULTS}

For the setup of physical configurations it is useful to recall the definitions of the dimensionless Rayleigh and Prandtl number which read

$$
\mathrm{Ra}=\frac{g \beta \Delta T H^{3}}{\nu \mathcal{D}}, \quad \operatorname{Pr}=\frac{\nu}{\mathcal{D}} .
$$

The characteristic velocity $U$ for thermal convection flows is generally set to the buoyancy velocity $U=\sqrt{g \beta \Delta T H}$, where $H$ denotes a problemdependent geometric height. A cell $(j, k)$ is flagged for refinement if any of the scaled gradient relations

$$
\begin{aligned}
& \left|\phi_{j+1, k}-\phi_{j, k}\right|>\epsilon_{\phi}, \quad\left|\phi_{j, k+1}-\phi_{j, k}\right|>\epsilon_{\phi}, \\
& \left|\phi_{j+1, k+1}-\phi_{j, k}\right|>\epsilon_{\phi}
\end{aligned}
$$

is satisfied for a particular macroscopic component $\phi_{j, k}$ and a prescribed limit $\epsilon_{\phi}$. If not stated otherwise, $\epsilon_{T}$ is set to $1 \%$ of maximum temperature and $\epsilon_{u}, \epsilon_{v}$ are set to $5 \%$ of characteristic velocity.

\section{A. Porous Plate}

In order to validate the basic numerical method, we use the problem of two porous plates with forced thermal convection also employed by Guo et al. [7]. This problem is set up as a Couette flow between two porous plates of which the upper is in motion. A constant flow is injected normal to the lower plate and leaves the domain through the top plate with the same rate. The bottom plate is cooled, while the upper plate is heated. The analytic solutions for the horizontal velocity and the temperature profile in steady state reads

$$
\begin{aligned}
u(y) & =U_{0}\left(\frac{e^{\operatorname{Re} \cdot y / H}-1}{e^{\operatorname{Re}}-1}\right), \\
T(y) & =T_{C}+\Delta T\left(\frac{e^{\operatorname{RePr} \cdot y / H}-1}{e^{\operatorname{RePr}}-1}\right),
\end{aligned}
$$



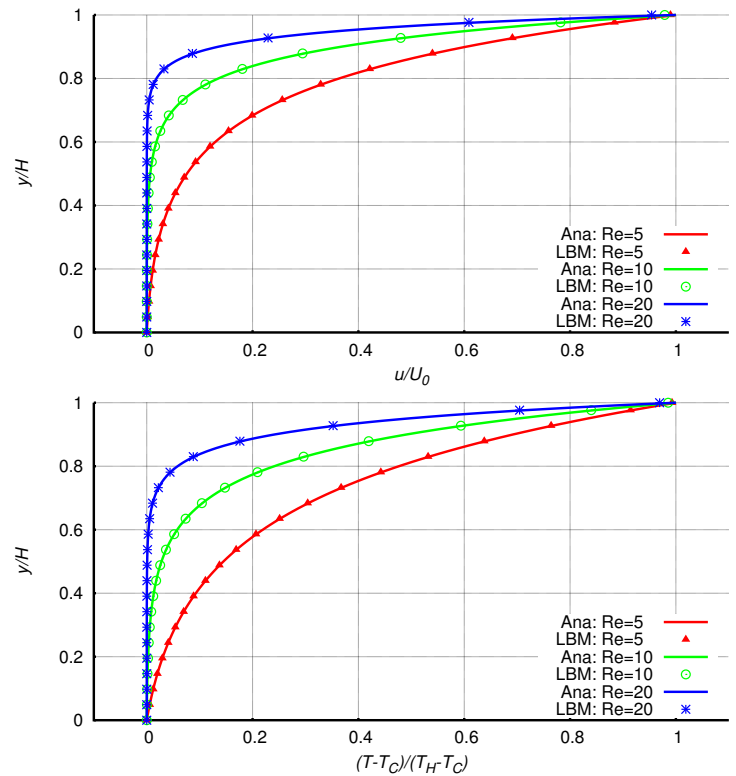

Fig. 3. Comparison of velocity and temperature distribution predicted for different Re in comparison with analytical solution

where $U_{0}$ is the velocity of the upper plate. The Reynolds number $R e$ is based on the injection velocity $V_{0}$ and is given by $\operatorname{Re}=\frac{V_{0} \cdot H}{\nu}$. We study three different configurations with varying Reynolds number. The Prandtl number ist fixed and set to $\operatorname{Pr}=0.71$, which corresponds to air. The Rayleigh number is set to $R a=100$. The velocity of the upper plate is also fixed and set to $U_{0}=0.1$. Finally, the dimension-less relaxation time $\tau_{\nu}$ on the coarsest level is prescribed as $\tau_{\nu}=1 / 1.25$. The simulations are performed for the Reynolds numbers $\operatorname{Re}=5,10$ and 20 are simulated using a base grid of $64 \times 32$ cells. Successive embedded static refinement with four additional levels with refinement factors $r_{1, \ldots 4}=4$ is realized in the region $[0,64] \times[22,32]$ (not specially visualized here). Since in this problem the velocity and temperature distributions are constant in the $x$-direction, we compare the numerical predictions with the analytic solution along the center line in the $y$ direction. Figure 3 plots the normalized numerical results vs. the analytical solutions. The agreement is obviously excellent. Also, the calculation of the L2-norm of the error between the computational and the analytical results shows deviations of at most $2 \%$ in all cases.

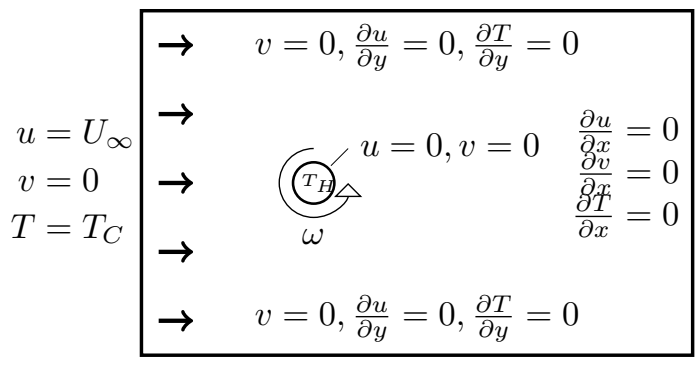

Fig. 4. Setup for the flow past the heated rotating cylinder
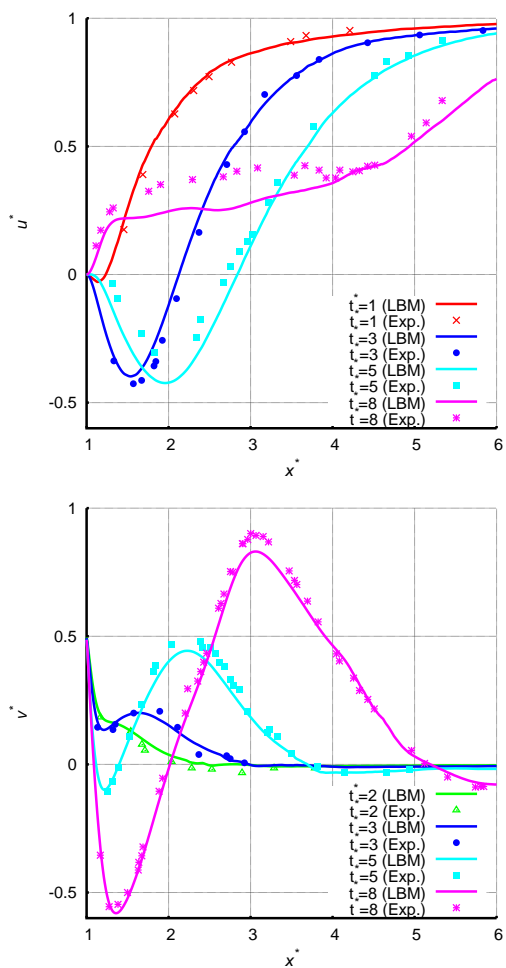

Fig. 5. Time evolution of the velocity components along the $\mathrm{x}$-axis for $\mathrm{Re}=200$ and $k=0.5$.

\section{B. Fluid flow past a heated rotating cylinder}

In order to test the dynamic adaptation capabilities and boundary conditions for embedded complex geometries, we study the setup of a twodimensional fluid flow past a heated isothermal rotating cylinder. The origin is located in the center of the cylinder. As shown in Figure 4, the left boundary is an inlet with constant temperature $T_{C}$, 


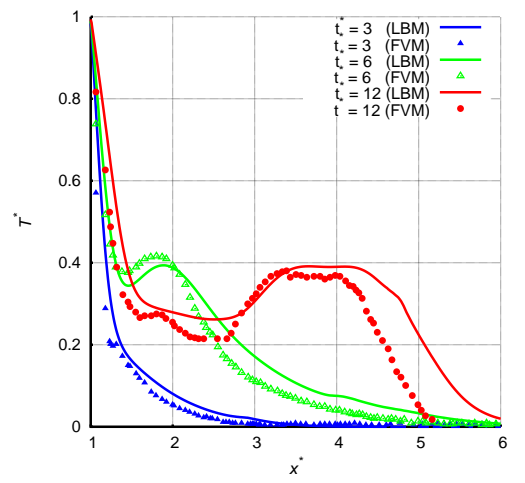

Fig. 6. Time evolution of the Temperature along the $\mathrm{x}$-axis for $\operatorname{Re}=200, \operatorname{Pr}=0.5$ and $k=0.5$.

zero vertical velocity and constant inflow velocity $U_{\infty}$. On the right hand side of the domain, an outlet is modeled by imposing zero horizontal gradient boundary conditions for velocity and temperature. Slip adiabatic wall boundary conditions are applied at the upper and lower boundary. The cylinder boundary is modeled as a no-slip wall, which is isothermally heated to the constant temperature $T_{H}$ and has the constant prescribed angular velocity $\Omega$. In terms of the cylinder radius $R=15$, the computational domain has the extensions $[-6 R, 16 R] \times$ $[-8 R, 8 R]$, which is sufficiently large to eliminate boundary influences on the solution [16]. A base grid of $288 \times 240$ cells is used and three additional levels refined by the factors $r_{1}=2$ for level 1 and $r_{2,3}=4$ for the other levels are applied. The dynamic refinement is based on scaled gradients of the velocity components as well as the temperature. The entire velocity field is initialized as $\left(U_{\infty}, 0\right)^{T}$ and the temperature field to the constant value $T_{C}$. The Reynolds number is given by $\operatorname{Re}=2 U_{\infty} R / \nu$ and is set to $\operatorname{Re}=200$, where $U_{\infty}=0.01$ is used. The peripheral velocity $V$ of the rotating cylinder is given by $V=\Omega R$. With the parameter $k=V / U_{\infty}=0.5$ prescribed, we can determine $V$ and the angular velocity $\Omega$. The Prandtl number is set to $\mathrm{Pr}=0.5$. To allow direct comparison to the experimental results by Coutanceau et al. [17] all variables are normalizing by using $R$ and $U_{\infty}$ as reference length and velocity, $\frac{T-T_{C}}{T_{H}-T_{C}}$ as reference temperature and the time normalization factor follows as $R / U_{\infty}$. Figure 5 compares the temporal evolution of the velocity components along rep-

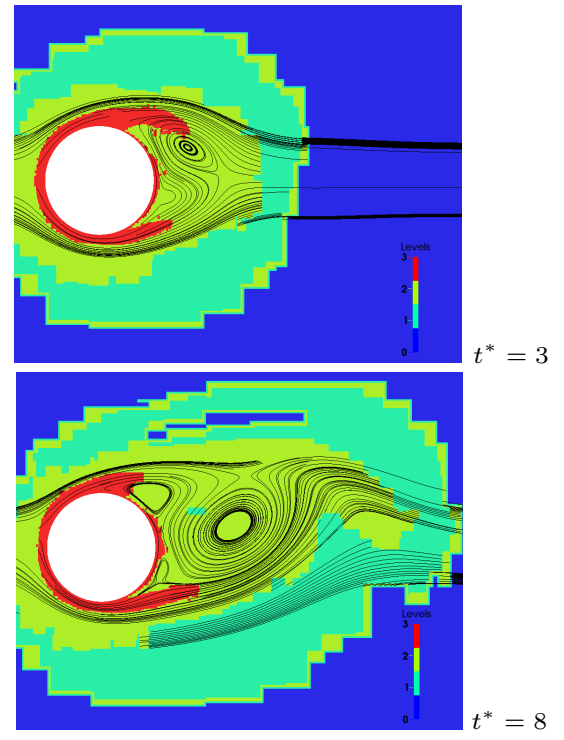

Fig. 7. Evolution of the velocity field and the adaptive mesh refinement regions for $\operatorname{Re}=200$ and $k=0.5$.

resentative points on the $x$-axis obtained in the simulation and with data from the experiment. Figure 6 displays the time evolution of the scalar temperature versus numerical results reported by Lai et al. [18] who adopted a finite volume method with non-orthogonal grids. Again, our simulation results are in good agreement. Finally, Fig. 7 shows the dynamic adaption during the computation by displaying streamlines and the domains of different mesh refinement levels. The onset of vortex shedding can be inferred.

\section{Natural convection in a square cavity}

In order to benchmark the overall method we employ a two-dimensional square cavity with differentially heated walls. The vertical walls are held at temperatures $T_{H}$ and $T_{C}$; adiabatic boundary conditions are applied at top and bottom. On all four walls we prescribe no-slip boundary conditions for the velocity field. Figure 8 depicts this setup. The flow has the Prandtl number $\operatorname{Pr}=$ 0.71 (air) and Rayleigh numbers $\mathrm{Ra}=10^{j}$ with $j=3, \ldots, 8$ and accordingly increasing velocity $U$ are studied. The reference temperature is given by $T_{r e f}=\left(T_{H}+T_{C}\right) / 2$. The simulations were terminated after reaching steady state. Two additional levels of refinement with $r_{1, \ldots, 2}=2$ are used 


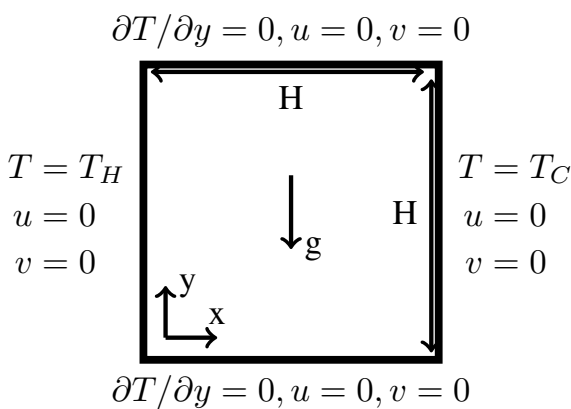

Fig. 8. Configuration of the two dimensional cavity

and the base mesh has $\left(H \Delta x_{0}\right)^{2}$ cells, whereby $\Delta x_{0}=1$ and $H$ is given in the left column of Table I.

We compare our adaptive simulation results to published reference data. Davis [19] solves the NS equations on a uniform square mesh by a finite difference method and uses Richardson extrapolation subsequently to obtain high accuracy results. Guo et al. [7] use the incompressible thermal LBGK approach presented above with a uniform mesh. Kuznik et al. [9] use a D2Q9 DDF LBM approach with non-uniform mesh resolution. Evaluated in Table I are the maximal horizontal velocity $u_{\max }$ along the vertical center line at $x=H / 2$ and the location $y_{\max }$ of its occurence and similarly for the horizontal center line at $y=H / 2$, the maximal vertical velocity $v_{\max }$ and its location $x_{\text {max }}$. Furthermore, the averaged Nusselt number is computed as

$$
\mathrm{Nu}_{\mathrm{ave}}=-\left.\int_{0}^{H} \frac{1}{\Delta T} \frac{\partial T}{\partial x}\right|_{x=0} d y .
$$

Velocity values in Table I are normalized by the reference velocity $\mathcal{D} / H$. As expected, $u_{\max }, v_{\max }$ and $\mathrm{Nu}_{\text {ave }}$ increase with increasing Rayleigh number Ra. Comparing the $\mathrm{Nu}$ numbers predicted by our method with the literature, an agreement within $2 \%$ is found for all $\mathrm{Ra}$ numbers. To give an impression of the flow solution, contours of the temperature fields and streamlines are presented in Fig. 9 for three considered Ra numbers. In the graph with the contours predicted for $\mathrm{Ra}=10^{7}$ the mesh refinement levels realized in the domain are additionally highlighted with colours. As can be
TABLE I. COMPARISON OF THE SIMULATION RESULTS: NATURAL CONVECTION IN THE SQUARE CAVITY

\begin{tabular}{|c|c|c|c|c|c|c|}
\hline & Ref. & $u_{\max }$ & $y_{\max }$ & $v_{\max }$ & $x_{\max }$ & $\mathrm{Nu}_{\text {ave }}$ \\
\hline $\begin{array}{l}\mathrm{Ra}=10^{3} \\
U=0.01 \\
H=100\end{array}$ & $\begin{array}{l}\mathrm{a} \\
\mathrm{b} \\
\mathrm{c} \\
\mathrm{d}\end{array}$ & $\begin{array}{l}3.640 \\
3.649 \\
3.655 \\
3.636\end{array}$ & $\begin{array}{l}0.810 \\
0.813 \\
0.813 \\
0.809\end{array}$ & $\begin{array}{l}3.688 \\
3.697 \\
3.699 \\
3.686\end{array}$ & $\begin{array}{l}0.180 \\
0.178 \\
0.180 \\
0.174\end{array}$ & $\begin{array}{l}1.115 \\
1.114 \\
1.115 \\
1.117\end{array}$ \\
\hline $\begin{array}{l}\mathrm{Ra}=10^{4} \\
U=0.02 \\
H=150\end{array}$ & $\begin{array}{l}\mathrm{a} \\
\mathrm{b} \\
\mathrm{c} \\
\mathrm{d}\end{array}$ & $\begin{array}{l}16.161 \\
16.178 \\
16.076 \\
16.167\end{array}$ & $\begin{array}{l}0.823 \\
0.823 \\
0.820 \\
0.821\end{array}$ & $\begin{array}{l}19.595 \\
19.617 \\
19.637 \\
19.597\end{array}$ & $\begin{array}{l}0.118 \\
0.119 \\
0.117 \\
0.120\end{array}$ & $\begin{array}{l}2.239 \\
2.245 \\
2.248 \\
2.246\end{array}$ \\
\hline $\begin{array}{l}\mathrm{Ra}=10^{5} \\
U=0.05 \\
H=200\end{array}$ & $\begin{array}{l}\mathrm{a} \\
\mathrm{b} \\
\mathrm{c} \\
\mathrm{d}\end{array}$ & $\begin{array}{l}34.666 \\
34.730 \\
34.834 \\
34.962\end{array}$ & $\begin{array}{l}0.855 \\
0.855 \\
0.859 \\
0.854\end{array}$ & $\begin{array}{l}68.457 \\
68.590 \\
68.267 \\
68.578\end{array}$ & $\begin{array}{l}0.066 \\
0.066 \\
0.062 \\
0.067\end{array}$ & $\begin{array}{l}4.504 \\
4.510 \\
4.535 \\
4.518\end{array}$ \\
\hline $\begin{array}{l}\mathrm{Ra}=10^{6} \\
U=0.05 \\
H=200\end{array}$ & $\begin{array}{l}\mathrm{a} \\
\mathrm{b} \\
\mathrm{c} \\
\mathrm{d}\end{array}$ & $\begin{array}{l}64.756 \\
64.630 \\
65.361 \\
64.133\end{array}$ & $\begin{array}{l}0.850 \\
0.850 \\
0.852 \\
0.860\end{array}$ & $\begin{array}{l}220.12: \\
219.360 \\
216.415 \\
220.537\end{array}$ & $\begin{array}{l}0.038 \\
0.038 \\
0.039 \\
0.038\end{array}$ & $\begin{array}{l}8.804 \\
8.806 \\
8.778 \\
8.792\end{array}$ \\
\hline $\begin{array}{l}\mathrm{Ra}=10^{7} \\
U=0.05 \\
H=256\end{array}$ & $\begin{array}{l}\mathrm{a} \\
\mathrm{d}\end{array}$ & $\begin{array}{l}140.25: \\
148.768\end{array}$ & $\begin{array}{l}0.887 \\
0.881\end{array}$ & $\begin{array}{l}702.45 ! \\
702.029\end{array}$ & $\begin{array}{l}0.021 \\
0.020\end{array}$ & $\begin{array}{l}16.429 \\
16.408\end{array}$ \\
\hline $\begin{array}{l}\mathrm{Ra}=10^{8} \\
U=0.05 \\
H=256\end{array}$ & $\begin{array}{l}\mathrm{a} \\
\mathrm{d}\end{array}$ & $\begin{array}{l}297.14 \\
321.457\end{array}$ & $\begin{array}{l}0.945 \\
0.940\end{array}$ & $\begin{array}{l}2228.4 \\
2243.36\end{array}$ & $\begin{array}{l}0.012 \\
0.012\end{array}$ & $\begin{array}{l}29.954 \\
29.819\end{array}$ \\
\hline
\end{tabular}

seen from the predominantly vertical isothermal, for small Ra the heat transfer is mainly controlled by conduction between the heated walls. For larger $\mathrm{Ra}$ the isotherms become more horizontal in the cavity center indicating increasing convection. Indicated by the closely spaced isothermals near the hot and cold wall, the decreasing thermal boundary layer thickness with increasing Rayleigh number is clearly reflected. It is in this region where on-thefly mesh resolution is particularly beneficial.

\section{CONCLUSIONS}

A 2D dynamically adaptive thermal lattice Boltzmann method has been developed. Successful validation against analytic solutions of the NavierStokes equations as well as verification by comparing our data with numerical results from the literature has been achieved. For the benchmark of a two-dimensional heated cavity the predictions are in good agreement with published results. A comprehensive analysis of CPU-time and memory savings by employing the adaptive method will be 


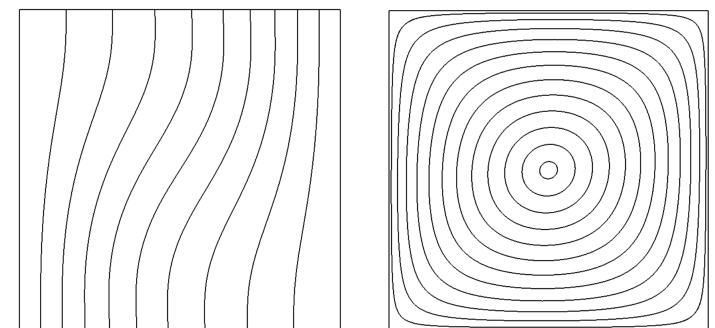

$\mathrm{Ra}=10^{3}$

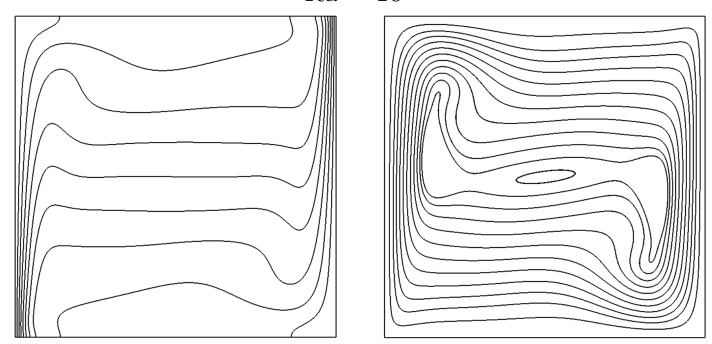

$\mathrm{Ra}=10^{6}$

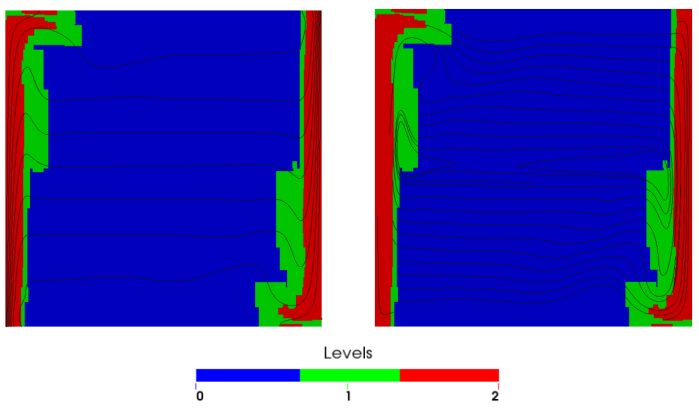

$$
\mathrm{Ra}=10^{7}
$$

Fig. 9. Simulation results of natural convective flow in the square cavity. Left: Contours of isotherms. Right: Streamlines.

conducted in the future. The extension to three space dimensions and validation for turbulent convective flows is ongoing.

\section{REFERENCES}

[1] S. Chen and G. Doolen, "Lattice Boltzmann method for fluid flows," Аnnu. Rev. Fluid Mech., vol. 30, pp. 329364, 1998.

[2] D. Hähnel, Molekulare Gasdynamik. Springer, 2004.

[3] X. He and L.-S. Luo, "Lattice Boltzmann model for the incompressible Navier-Stokes equation," J. Stat. Phys., vol. 88, pp. 927-944, 1997.

[4] Y. Qian, D. D'Humires, and P. Lallemand, "Lattice BGK models for Navier-Stokes equation," Europhys. Lett., vol. 17, p. 479, 1992.
[5] X. He, S. Chen, and G. Doolen, "A novel thermal model for the lattice Boltzmann method in incompressible limit," J. Comput. Phys., vol. 146, pp. 282-300, 1998.

[6] Y. Qian, "Simulating thermohydrodynamics with lattice BGK models," Journal of Scientific Computing, vol. 8(3), pp. 231-241, 1993.

[7] Z. Guo, B. Shi, and C. Zheng, "A coupled lattice BGK model for the Boussinesq equations," Int. J. Numer. Meth. Fluids, vol. 39, pp. 325-342, 2002.

[8] Z. Yu and L.-S. Fan, "An interaction potential based lattice Boltzmann method with adaptive mesh refinement (AMR) for two-phase flow simulation," J. Comput. Phys., vol. 17, pp. 6456-6478, 2009.

[9] F. Kuznik, J. Vareilles, G. Rusaouen, and G. Kraiss, "A double-population lattice Boltzmann method with nonuniform mesh for the simulation of natural convection in a square cavity," Int. J. Heat Fluid Fl., vol. 28, pp. 862-870, 2007.

[10] R. Deiterding, "Block-structured adaptive mesh refinement - theory, implementation and application," ESAIM Proc., vol. 34, pp. 97-150, 2011.

[11] N.-Z. He, N.-C. Wang, B.-C. Shi, and Z.-L. Guo, "A unified incompressible lattice BGK model and its application to three-dimensional lid-driven cavity flow," Chinese Phys., vol. 13, 2004.

[12] P. Bhatnagar, E. Gross, and M. Krook, "A model for collisional processes in gases I: small amplitude processes in charged and in neutral one-component systems," Phys. Rev., vol. 94, pp. 511-525, 1954.

[13] A. Mohamad and A. Kuzmin, "A critical evaluation of force term in lattice Boltzmann method, natural convection problem," Int. J. Heat Mass Transfer, vol. 53, pp. 990-996, 2010.

[14] M. Berger and P. Colella, "Local adaptive mesh refinement for shock hydrodynamics," J. Comput. Phys., vol. 82, pp. 64-84, 1988.

[15] H. Chen, O. Filippova, J. Hoch, K. Molvig, R. Shock, C. Teixeira, and R. Zhang, "Grid refinement in lattice Boltzmann methods based on volumetric formulation," Physica A, vol. 362, pp. 158-167, 2006.

[16] Y. Yan and Y. Zu, "Numerical simulation of heat transfer and fluid flow past a rotating isothermal cylinder - A LBM approach," Int. J. Heat Mass Transfer, vol. 51, pp. 2519-2536, 2008

[17] M. Coutanceau and C. Menard, "Influence of rotation on the near-wake development behind an impulsively started circular cylinder," J. Fluid Mech., vol. 158, pp. 399-446, 1985.

[18] H. Lai and Y. Yan, "The effect of choosing dependent variables and cell-face velocities on convergence of the SIMPLE algorithm using non-orthohonal grids," Int. J. Numer. Method H., vol. 11, pp. 524-546, 2001.

[19] G. De Vahl Davis, "Natural convection of air in a square cavity a benchmark numerical solution," Int. J. Numer. Meth. Fluids, vol. 3, pp. 249-264, 1983. 